\title{
Riesgos psicosociales y desempeño laboral de los funcionarios del Hospital Básico Píllaro y su dirección distrital
}

\author{
Fecha de recepción: 30-12-2020 • Fecha de aceptación: 28-01-2021 • Fecha de publicación: 10-02-2021
}

Iván Danilo Galarza Torresano'

ivan.galarza@iste.edu.ec Instituto Tecnológico Superior Universitario España, Ecuador https://orcid.org/0000-0002-4606-2333

Blanca Trinidad Tocto Villarreal ${ }^{2}$ blanca.tocto@iste.edu.ec Instituto Tecnológico Superior Universitario España, Ecuador https://orcid.org/0000-0002-9968-9930

Byron Calixto Peñaloza Montenegro ${ }^{3}$ byron.penaloza@iste.edu.ec Instituto Tecnológico Superior Universitario España, Ecuador https://orcid.org/0000-0001-9943-1350

\section{RESUMEN:}

Los cambios acelerados en el mundo actualmente han generado un impacto directo en el ámbito laboral, la falta de preparación a esos cambios inesperados impacta también en el comportamiento y salud mental de los trabajadores; por tal motivo, el objetivo del presente estudio fue determinar la influencia de los riesgos psicosociales en el desempeño laboral de los funcionarios del Hospital Básico del Cantón Píllaro y su Distrito 18D05. El resultado del análisis, una vez aplicados los instrumentos de medición, identifica que el principal problema fue el inadecuado manejo de los riesgos psicosociales en los funcionarios a causa de los altos niveles de carga laboral y las exigencias por parte de los niveles superiores, la asignación de obligaciones laborales en horarios fuera de trabajo, el desconocimiento de herramientas metodológicas en el manejo de riesgos psicosociales, el apoyo reducido de los niveles superiores y compañeros, además de la inseguridad en el empleo. La investigación tiene 
un enfoque mixto cuali-cuantitativo, con carácter de tipo descriptivo y correlacional; la toma de datos se realizó mediante un cuestionario estructurado en dos partes: riesgos psicosociales y desempeño, a su vez se aplicó el método estadístico Alpha de Cronbach para analizar la correlación de las variables, y para tabular y procesar los datos se utilizó la herramienta SPSS.

\title{
PALABRAS CLAVE: riesgos psicosociales, salud mental, carga laboral, Alpha de Cronbach.
}

\begin{abstract}
The accelerated changes in the world today have generated a direct impact on the workplace, the lack of preparation for these unexpected changes also impacts on the behavior and mental health of workers; therefore, the objective of this study was to determine the influence of psychosocial risks on the work performance of employees of the Hospital Básico del Cantón Píllaro and its District 18D05. The result of the analysis, once the measurement instruments were applied, identifies that the main problem was the inadequate management of psychosocial risks in the employees due to the high levels of workload and the demands of higher levels, the assignment of work obligations in non-working hours, the lack of knowledge of methodological tools in the management of psychosocial risks, the reduced support from higher levels and colleagues, in addition to job insecurity. The research has a mixed qualitative-quantitative approach, with a descriptive and correlational nature; data collection was carried out by means of a questionnaire structured in two parts: psychosocial risks and performance, and the Cronbach Alpha statistical method was applied to analyze the correlation of variables, and the SPSS tool was used to tabulate and process the data.
\end{abstract}




\section{Introducción}

En la actualidad, los rápidos cambios generados por una economía global, la crisis mundial que afecta el empleo y la competitividad, producen un impacto directo en el ambiente laboral y sus integrantes; eso sumado a la falta de cultura organizacional que entre otros aspectos brinda poca atención a las necesidades del talento humano ocasionan serios problemas relacionados a la salud mental, así como también al comportamiento y desempeño de las personas, siendo por tanto causales del presente estudio.

Ajustarse a estas transformaciones implica sujetarse a nuevos y complejos procesos, modificar conocimiento, destrezas, habilidades, horarios de trabajo, tiempo para la familia, etc., así como la lucha permanente por mantener un puesto de trabajo. El sector público no es la excepción, por el contrario, debido a las características y estructura de su sistema, las organizaciones que laboran dentro de este ámbito están expuestas a diferentes situaciones e inconvenientes que permanentemente requieren atención; las instituciones de salud pública están relacionadas directamente por este fenómeno, ya que al depender de un presupuesto estatal, así como de regulaciones y normativas, sumado al avance tecnológico y a la delicada labor de proveer atención médica, el desafío y obligaciones para el personal de la salud dentro de las diferentes áreas se multiplica y se torna por demás complejo. La situación política es otro aspecto a considerar que provoca también inestabilidad laboral dentro de este sector, convirtiéndose también en otro elemento de presión psicológica permanente que puede impactar en su condición física y comportamental,

Según la Organización Mundial de la Salud (2017) y la Organización Internacional del Trabajo (OIT), las organizaciones deben precautelar la salud mental de sus trabajadores. La constitución ecuatoriana también señala mediante el acuerdo ministerial MDT-2017-0082 emitido el 19 de septiembre del 2017 su decisión de erradicar elementos relacionados a esta problemática en los trabajadores y la obligatoriedad de implementar un programa que permita evaluar, controlar y erradicar los riesgos psicosociales.

En Ecuador, el Ministerio de Trabajo, conjuntamente con el Ministerio de Salud Pública, serían los órganos responsables de hacer que se cumpla la normativa direccionada a velar por la salud y bienestar del personal de esta área y dentro de este contexto, quienes tienen la obligación de sancionar conforme la legislación y las leyes.

Si en los hospitales se genera un ambiente adverso, éste debe ser canalizado de forma eficiente y conforme a las normativas por el personal responsable, debido a que por lo general los trabajadores están preparados para ejercer su profesión, más no para manejar adecuadamente los fenómenos del estrés que influye y afecta en su condición humana para el logro de sus objetivos, tanto a nivel personal, profesional e institucional.

Sin embargo, aún existe desconocimiento de herramientas metodológicas y falta de aplicación de la normativa para el manejo de riesgos psicosociales por parte de líderes y autoridades que buscan cualquier otra justificación a la falta de productividad y cumplimiento de objetivos contribuyendo a generar un ambiente cargado de tensión entre otros aspectos que ya han dado 
lugar a probables enfermedades derivadas del estrés laboral.

De no atenderse esta necesidad de forma adecuada, y al ser repetitivas y permanentes las cargas emocionales, podrían dar lugar a daños temporales y/o permanentes en la condición física y mental de cada trabajador, y con esto lógicamente a la disminución de su rendimiento; por lo mismo y debido a que el principal problema encontrado en el estudio fue el inadecuado manejo de los riesgos psicosociales en los funcionarios a causa de los altos niveles de exigencia laboral, generado por los niveles superiores refiriéndonos a todos y cada uno de los puestos de trabajo así como a la asignación de obligaciones laborales en horarios fuera de trabajo, el objetivo del presente estudio fue determinar la influencia de los riesgos psicosociales en el desempeño laboral de los funcionarios del Hospital Básico del Cantón Píllaro y el Distrito 18D05.

Para el estudio se consideran las dimensiones psicosociales: exigencias psicológicas, control sobre el trabajo, compensación laboral, apoyo social y calidad de liderazgo, trabajo familia y capital social.

\subsection{Los riesgos psicosociales}

Se puede manifestar que los riesgos psicosociales y el estrés laboral son problemas que más dificultades presentan en el ámbito de la seguridad y salud en el trabajo según la Agencia Europea para la Seguridad y Salud en el Trabajo. Además, se considera que los riesgos psicosociales son la causa de más accidentes y enfermedades laborales y según la VI encuesta de condiciones de trabajo en España realizada por el Instituto Nacional de Seguridad e Higiene en el Trabajo, el $70,9 \%$ de los encuestados manifiesta estar expuesto a accidentes de trabajo, siendo un porcentaje más elevado el caso del personal de la salud (74,9\%) debido a que los cortes y pinchazos se producen por falta de atención o distracciones. Además la carga física, mental o repetitiva de trabajo da muestras de síntomas psicosomáticos como los dolores de cabeza, mareos, cansancio, sueño, que pueden influir negativamente en la salud física y mental del personal del hospital, causantes de los riesgos Psicosociales. El dolor de espalda, cuello y estrés tanto para varones como para mujeres son los problemas más recurrentes según la encuesta seguidos por cansancio, alteraciones de sueño y cefaleas. "En el 2014, la Organización Internacional del Trabajo calculó que los accidentes y las enfermedades profesionales causan más de 2,3 millones de muertes anuales, de las cuales más de 350.00 son por accidentes de trabajo, y aproximadamente 2 millones son por enfermedades profesionales" (Red Regional de Bibliotecas OIT para América Latina y el Caribe, 2017).

En países de la región como Perú, Chile y Ecuador se advierte sobre el aparecimiento de este tipo de riesgos, también denominados "riesgos psicosociales emergentes" debido a que se originan en la globalización y su impacto por medio de los cambios tecnológicos que se generan, y a la vez ponen en alerta a los países en vías de desarrollo y a sus organizaciones quienes con marcada desventaja se ven obligados a competir y defenderse, incrementando a sus exigencias ya existentes (Gil, 2012). La constitución ecuatoriana según el artículo № 461 del 15 de noviembre del 2004 publicado en el registro oficial emite el Instrumento Andino de Seguridad y Salud en el Trabajo que dispone la obligatoriedad de tomar medidas para disminuir los riesgos 
laborales, haciendo énfasis a los riesgos psicosociales. En Ecuador, algunas investigaciones a varios centros hospitalarios incluyen hallazgos importantes en los encuestados referente a su bienestar psicosocial laboral, debido a las grandes transformaciones sociales, demográficas y epidemiológicas en el perfil de la población generando nuevas exigencias y desafíos para los trabajadores del sector que a su vez afectan sus perfiles de salud, comportamiento y desempeño laboral. Todos estos problemas se agudizan debido a que los profesionales piensan que sus trabajos no son bien remunerados, por lo cual optan por renunciar (Acevedo, 2013).

De acuerdo con el Ministerio de Salud Pública (2021), la población de usuarios es alta y los servicios que brinda se organizan por su complejidad, es así que se beneficia de atención médica gratuita suma aproximadamente 38.239 habitantes y a pesar de su vulnerabilidad la Dirección Provincial de Salud de Tungurahua (2021) no dispone de estadísticas relacionadas a la salud laboral. Esto causa serios problemas porque genera equivocaciones, lo que agudiza el estrés laboral del personal del hospital, que afecta directamente a sus pacientes.

\subsection{El desempeño laboral}

La afectación de un ambiente laboral deficiente y estresante en la condición mental y física de una persona puede producir profundas alteraciones en su comportamiento en general que están relacionados con la tensión nerviosa, decaimiento, falta de concentración, alteraciones cardíacas, irritabilidad, disminución en su autoestima, ansiedad, etc., aspectos que muy probablemente son causales indirectas como son la inasistencia, abandono del trabajo, dificultad en la toma de decisiones y disminución del rendimiento laboral.

De ahí que el desempeño laboral tiene una importante relación con el bienestar físico y mental del trabajador conforme lo demuestran los estudios realizados.

\section{Metodología}

La investigación tiene enfoque cualitativo porque se basa en las características de las variables y se fundamentan en una realidad subjetiva, esta investigación fue de características transversales (Salgado, 2007), porque se realizó en una delimitación temporal determinada, además la información se recogió por medio de la investigación de campo a las personas, además se investigó por medio de fuentes de consulta secundarias que sirvieron de sustento para el respectivo procesamiento e interpretación. Se manifiesta que también se usó el enfoque cuantitativo, porque se realizó cálculos matemáticos y se tabulo por medio de la hoja de cálculo de Excel.

\subsection{Población}

La población corresponde a 170 funcionarios del Hospital Básico Píllaro (2021y su Dirección Distrital también conocida como Distrito de Salud 18D05 Santiago de Píllaro (Dirección Distrital 18D05-Santiago de Pillaro - Salud, 2021); quienes integran el personal son: administrativo (directivos y personal técnico y de apoyo) y hospitalario (médicos, enfermeras y personal 
asistencial), tal como se refleja en la Tabla 1. La institución se conforma por 1 hospital básico, 1 unidad anidada, la sede distrital, las 9 unidades operativas entre centros y sub-centros de salud del cantón Píllaro. Es parte de la zona 3 del Ministerio de Salud Pública a través de la Dirección de Salud de Tungurahua.

Tabla 1.

Población trabajadores

\begin{tabular}{|c|c|c|}
\hline NIVELES & $\begin{array}{l}\text { DISTRIBUCIÓN DE } \\
\text { LA POBLACIÓN }\end{array}$ & PORCENTAJE \\
\hline $\begin{array}{l}\text { Administrativo } \\
\text { Dirección } \\
\text { hospitalaria distrital }\end{array}$ & 33 & $19.41 \%$ \\
\hline $\begin{array}{l}\text { Operativo } \\
\text { Hospital básico/ } \\
\text { unidades }\end{array}$ & 177 & $80.59 \%$ \\
\hline TOTAL & 170 & $100 \%$ \\
\hline
\end{tabular}

Fuente: Datos Distrito 18D05 - Pillaro Salud

\subsection{Muestra}

La muestra no hubo necesidad de extraerla debido a que las autoridades facilitaron las herramientas y logística para la realización del estudio, por lo tanto, se realizó el análisis con todos los elementos disponibles

Se aplicó el método estadístico Alpha de Cronbach (Ruiz, 2021) para analizar la correlación de las variables, y para tabular y procesar los datos se utilizó la herramienta SPSS que a su vez permitió realizar el análisis factorial y de regresión lineal comprobando así la hipótesis de investigación. Finalmente se elaboró una guía con el fin de contribuir en las decisiones de intervención.

\subsection{Variables a las que se aplicó el método del Alfa de Cronbach:}

- Riesgos psicosociales

- Desempeño laboral

Se aplicaron los cuestionarios de riesgos psicosociales y desempeño laboral posterior a la realización de una prueba piloto a 25 integrantes de la plantilla del Hospital Básico Píllaro y el Distrito 18D05 que constituyen aproximadamente el 15\% de colaboradores; se procedió entonces a aplicar el Alfa de Cronbach con la finalidad de comprobar la consistencia y validez del cuestionario de evaluación de riesgos psicosociales y desempeño laboral integrado por un total de 45 ítems (38 ítems que miden el aspecto psicosocial y 7 ítems que se refieren al desempeño laboral) con el apoyo de la herramienta estadística SPSS. Como referencia, la fiabilidad del índice alfa debe medir 
entre 0.7 a 0.95; los ítems miden un mismo constructo en escala tipo Likert, asumiendo su alta correlación; este es uno de los métodos más utilizados para medir la confiabilidad a través de la consistencia interna del instrumento.

En cuanto a los resultados de la correlación de las variables descritas, se presenta en la Tabla 2 un resumen.

Tabla 2.

Resumen del procesamiento de casos

\begin{tabular}{|l|l|l|l|}
\hline \multicolumn{2}{|c|}{} & N & $\%$ \\
\hline \multirow{4}{*}{ Casos } & Valido & 25 & 100 \\
& & & \\
\cline { 2 - 5 } & Excluido & 0 & 0 \\
\cline { 2 - 4 } & Total & 25 & 100 \\
\hline
\end{tabular}

La eliminación por lista se basa en todas las variables del procedimiento

Fuente: elaboración propia

No se excluye ningún valor por lo que la información de los 25 encuestados se valida con el $100 \%$ de elementos.

Por otra parte, en cuanto a las estadísticas de fiabilidad, en la Tabla 3 se muestra una representación.

Tabla 3.

Estadísticas de fiabilidad

\begin{tabular}{|l|l|}
\hline Alfa de Cronbach & N de elementos \\
\hline .811 & 45 \\
& \\
\hline
\end{tabular}

Fuente: Datos de la encuesta (SPSS)

De esta manera, se confirma la fiabilidad de los 45 ítems del instrumento para evaluar los riesgos psicosociales y el desempeño laboral. El resultado ha sido un coeficiente alfa de 0,811, es decir superior a .8, por lo cual existe una muy buena correlación entre las variables; riesgos psicosociales y desempeño laboral.

\section{Resultados}

Cualquier proceso de evaluación se debe ajustar a lo establecido en la Ley, tales como el Acuerdo Ministerial $N^{\circ} 82$ del registro oficial del 16 de junio del 2017, a efectos de erradicar la discriminación en el ámbito laboral, así como además al artículo 33 de la Constitución aprobada 
en el 2008, donde se contempla la obligatoriedad de promover la salud de los trabajadores; dentro de las acciones a seguir está el identificar, evaluar, prevenir, corregir y controlar los riesgos psicosociales.

El cuestionario buscará identificar y medir los factores de riesgo psicosocial, en base a las características de la organización que tienen evidencia científica de su afectación a la salud. Este integra las dimensiones más aceptadas y aplicadas en la mayoría de encuestas internacionalmente como son: cincuenta y cinco exigencias psicológicas laborales, trabajo activo y desarrollo de habilidades, apoyo social en la empresa y calidad del liderazgo, compensaciones, doble presencia y capital social, además de sub-dimensiones. Contiene 38 preguntas sobre condiciones de trabajo, exposición a factores psicosociales, salud y bienestar personal.

\subsection{La evaluación del desempeño en el sector público}

El formulario SENRES- EVAL-01 cumple con las especificaciones legales requeridas para el manejo del subsistema de evaluación del desempeño y es el principal instrumento dentro del proceso de medición que utiliza el Sector Público (Secretaría Nacional Técnica de Desarrollo de Recursos Humanos y Remuneración del Sector Público, 2007). El mismo se encuentra dividido en bloques o secciones y mide las siguientes dimensiones: indicador de gestión, competencias técnicas, competencias universales, iniciativa y trabajo en equipo, conocimiento, además del liderazgo y finalmente las sanciones por incumplimiento.

A continuación, se detalla la forma como son calificados los factores que componen el formulario en el que se asignan distintas ponderaciones que pueden llegar a sumar hasta 100\% (incluso hasta el $104 \%$ de existir adelanto de trabajo respecto al período subsiguiente).

- Indicador de gestión

Columna de cumplidos.- Integra las actividades, indicadores y metas del puesto. \% de cumplimiento. - Los resultados se ingresan en esta columna en porcentajes.

El nivel de cumplimiento se distribuye como se evidencia en la Tabla 4:

Tabla 4.

Resumen de los resultados del análisis del cumplimiento

\begin{tabular}{|l|l|l|l|}
\hline Ponderación & Descripción & Cuantitativa & Cualitativa \\
\hline 5 & Cumplimiento alto & $\geq 90,5 \%$ y el $100 \%$ & Excelente \\
\hline 4 & Cumplimiento mejor al esperado & $\geq 80,5 \%$ y el $90,40 \%$ & Muy bueno \\
\hline 3 & Cumplimiento esperado & $\geq 70,5 \%$ y el $80,40 \%$ & Satisfactorio \\
\hline 2 & Cumplimiento bajo al esperado & $\geq$ el 60,5 y el $70,4 \%$ & Deficiente \\
\hline 1 & Cumplimiento muy bajo al esperado & $\leq 60,4 \%$ & Inaceptable \\
\hline
\end{tabular}

Fuente: Tocto, Blanca (2018) con información de la Secretaría Nacional Técnica de Desarrollo de Recursos Humanos y Remuneración del Sector Público (2008) 


\section{- Conocimientos}

El porcentaje que si está asignado a este campo es el 8\% (Tabla 5).

Tabla 5.

Resumen de los resultados alcanzado en la dimensión del conocimiento

\begin{tabular}{|l|l|l|l|}
\hline Ponderación & Descripción & Cuantitativa & Cualitativa \\
\hline 5 & Conocimiento alto & $\geq 90,5 \%$ y el $100 \%$ & Sobresaliente \\
\hline 4 & Conocimiento mejor al esperado & $\geq 80,5 \%$ y el $90,40 \%$ & Muy bueno \\
\hline 3 & Conocimiento esperado & $\geq 70,5 \%$ y el $80,40 \%$ & Bueno \\
\hline 2 & Conocimiento bajo al esperado & $\geq$ el 60,5 y el $70,4 \%$ & Regular \\
\hline 1 & Conocimiento muy bajo al esperado & $\leq 60,4 \%$ & Insuficiente \\
\hline
\end{tabular}

Fuente: Tocto, Blanca (2018) con información de la Secretaría Nacional Técnica de Desarrollo de Recursos Humanos y Remuneración del Sector Público (2008)

\section{- Competencias técnicas}

El porcentaje asignado a este campo es del 8\% (Tabla 6).

Tabla 6.

Resumen de los resultados alcanzado en la dimensión de competencias técnicas

\begin{tabular}{|l|l|l|l|}
\hline Ponderación & Descripción & Cuantitativa & Cualitativa \\
\hline 5 & Desarrollo alto & $\geq 90,5 \%$ y el $100 \%$ & Altamente desarrollada \\
\hline 4 & Desarrollo mejor al esperado & $\geq 80,5 \%$ y el $90,40 \%$ & Desarrollada \\
\hline 3 & Desarrollo esperado & $\geq 70,5 \%$ y el $80,40 \%$ & Medianamente desarrollada \\
\hline 2 & Desarrollo bajo al esperado & $\geq$ el 60,5 y el $70,4 \%$ & Poco desarrollada \\
\hline 1 & Desarrollo muy bajo al esperado & $\leq 60,4 \%$ & No desarrollada \\
\hline
\end{tabular}

Fuente: Tocto, Blanca (2018) con información de la Secretaría Nacional Técnica de Desarrollo de Recursos Humanos y Remuneración del Sector Público (2008).

\section{- Competencias universales}

Está enfocada en la puesta en práctica de las destrezas, conocimientos y actitudes que se miden respecto a su relevancia en campos predeterminados del formulario. Se evalúa sobre el $8 \%$ (Tabla $7)$. 
Tabla 7.

Resumen de los resultados alcanzado en la dimensión de competencias universales

\begin{tabular}{|l|l|l|l|}
\hline Ponderación & Descripción & Cuantitativa & Cualitativa \\
\hline 5 & Frecuencia alta & $\geq 90,5 \%$ y el $100 \%$ & Siempre \\
\hline 4 & Frecuencia mejor ala esperado & $\geq 80,5 \%$ y el $90,40 \%$ & Frecuentemente \\
\hline 3 & Frecuencia esperado & $\geq 70,5 \%$ y el $80,40 \%$ & Alguna vez \\
\hline 2 & Frecuencia baja al esperada & $\geq$ el 60,5 y el $70,4 \%$ & Rara vez \\
\hline 1 & Frecuencia muy baja a la esperada & $\leq 60,4 \%$ & Nunca \\
\hline
\end{tabular}

Fuente: Tocto, Blanca (2018) con información de la Secretaría Nacional Técnica de Desarrollo de Recursos Humanos y Remuneración del Sector Público (2008).

- Trabajo en equipo e iniciativa

Este campo se evalúa sobre el $16 \%$, es decir a 5,33\% c/u, pero sí únicamente se evalúan trabajo en equipo e iniciativa a cada uno le corresponde el $8 \%$.

Tabla 8.

Resumen de los resultados alcanzado en la dimensión de trabajo en equipo, iniciativa y li derazgo

\begin{tabular}{|c|c|c|c|}
\hline Ponderación & Descripción & Cuantitativa & Cualitativa \\
\hline 5 & Frecuencia alta & $\geq 90,5 \%$ y el $100 \%$ & Siempre \\
\hline 4 & Frecuencia mejor a la esperado & $\geq 80,5 \%$ y el $90,40 \%$ & Frecuentemente \\
\hline 3 & Frecuencia esperado & $\geq 70,5 \%$ y el $80,40 \%$ & Alguna vez \\
\hline 2 & Frecuencia baja al esperada & $\geq$ el 60,5 y el $70,4 \%$ & Rara vez \\
\hline 1 & Frecuencia muy baja a la esperada & $\leq 60,4 \%$ & Nunca \\
\hline
\end{tabular}

Fuente: Tocto, Blanca (2018) con información de la Secretaría Nacional Técnica de Desarrollo de Recursos Humanos y Remuneración del Sector Público (2008).

\section{- Liderazgo}

Este se aplica a los puestos que tienen a su cargo la responsabilidad de manejo de personal. 
Tabla 9.

Resumen de los resultados alcanzado en la dimensión de liderazgo

\begin{tabular}{|c|c|c|c|}
\hline \multicolumn{4}{|l|}{ ESCALA } \\
\hline Ponderación & Descripción & Cuantitativa & Cualitativa \\
\hline 5 & Frecuencia alta & $\geq 90,5 \%$ y el $100 \%$ & Siempre \\
\hline 4 & Frecuencia mejor a la esperado & $\geq 80,5 \%$ y el $90,40 \%$ & Frecuentemente \\
\hline 3 & Frecuencia esperado & $\geq 70,5 \%$ y el $80,40 \%$ & Alguna vez \\
\hline 2 & Frecuencia baja al esperada & $\geq$ el 60,5 y el $70,4 \%$ & Rara vez \\
\hline 1 & Frecuencia muy baja a la esperada & $\leq 60,4 \%$ & Nunca \\
\hline
\end{tabular}

Fuente: Tocto, Blanca (2018) con información de la Secretaría Nacional Técnica de Desarrollo de Recursos Humanos y Remuneración del Sector Público (2008)

De los datos observados, los niveles de desempeño y cada una de sus dimensiones se encuentran entre una frecuencia esperada (conocimiento, iniciativa, trabajo en equipo, y liderazgo) a mejor de la esperada (desempeño, competencias técnicas y universales). En ninguno de los casos se presenta una frecuencia alta para cada una de las dimensiones. En lo concerniente a multas por incumplimiento, si bien el porcentaje en que aparecen es mínimo (3,53\%), es un valor importante de información ya que cada queja contra un funcionario o servidor público le restará el $4 \%$ de la calificación total obtenida. Sus deberes y obligaciones estarán sujetos a lo que la ley establece.

En la Tabla 10 se presenta un resumen de ponderaciones por factor.

Tabla 10.

Factores que componen el formulario aplicado

\begin{tabular}{|l|l|}
\hline Factores & Ponderación (\%) \\
\hline Indicadores de gestión del puesto & 60 \\
\hline Conocimientos & 8 \\
\hline Competencias técnicas del puesto & 8 \\
\hline Competencias Universales & 8 \\
\hline Trabajo en equipo, iniciativa y liderazgo & 16 \\
\hline Total & 100 \\
\hline
\end{tabular}

Fuente: Red Norma técnica del subsistema de evaluación del desempeño Art. 17 
Y en la Tabla 11 se muestra un resumen total del modelo aplicado.

Tabla 11.

Resumen del modelo

\begin{tabular}{|l|l|l|l|l|l|}
\hline Modelo & $\mathbf{R}$ & R cuadrado & $\begin{array}{l}\text { R cuadrado } \\
\text { ajustado }\end{array}$ & $\begin{array}{l}\text { Error } \\
\text { estándar de la } \\
\text { estimación }\end{array}$ & $\begin{array}{l}\text { Wurbin- } \\
\text { Watson }\end{array}$ \\
\hline 1 & $.773_{\mathrm{a}}$ & .598 & .596 & 1.52330 & 1.083 \\
\hline
\end{tabular}

Predictores: (Constante), Riesgo Psicosocial

Variable dependiente: Desempeño Laboral

Los resultados muestran en la tabla el valor de $R 2=0,598$ y la teoría señala que si el $R$ cuadrado tiene un valor cercano a 0 significa que el modelo no se ajusta bien a los datos; mientras que si va de 0,5 a 1 tiene bondad en su ajuste de tal forma que el modelo de regresión lineal simple explica adecuadamente la relación de riesgos psicosociales con el desempeño laboral detallando el nivel de variabilidad de la variable dependiente $60 \%$ mientras que el $40 \%$ queda explicada por otros factores. $R=0,773$ mientras más se acerca a 1 la correlación lineal es buena con lo que se cumple el criterio de linealidad además de el de normalidad de residuos ya que tiene cierta semejanza a una curva normal según se observa en el histograma de frecuencia.

\section{Conclusiones}

En cuanto a las conclusiones de los estudios realizados nos permiten mencionar que se verifica que el objetivo al determinar la influencia de los riesgos psicosociales en el desempeño laboral de los funcionarios del Hospital Básico Píllaro y su Dirección Distrital 18D05, una vez que las cargas factoriales mostraran un mayor peso del comportamiento de las medidas de influencia de las dos variables. La investigación es idónea y sobre estos resultados es que se diseñará el modelo de propuesta.

A su vez, se sustenta la investigación con suficiente fundamento científico, con lo que se contribuye a facilitar información valiosa para autoridades e involucrados en los que permite visualizar la necesidad y relevancia de la implementación de un plan de prevención de riesgos psicosociales en sus organizaciones.

Se cumple con el análisis de los niveles de riesgos psicosociales y el desempeño laboral de los funcionarios en el Hospital Básico Píllaro y su Dirección Distrital, luego se determinó la situación de la institución frente a las variables investigadas.

El resultado mostró que las dimensiones de exposición psicosocial con mayor afectación fueron: exigencias psicológicas con $62 \%$; control sobre el trabajo con $51,60 \%$; compensación laboral con $62,80 \%$; apoyo social y calidad de liderazgo con $59,80 \%$ y capital social con $63,50 \%$; se concluyó que el nivel de riesgo al que están expuestas es intermedio.

De esta manera, dentro de estas 141 dimensiones, se encontraron 11 factores de riesgos 
psicosocial a tomarse en cuenta ya que mostraron un nivel de riesgo intermedio, estas fueron: ritmo de trabajo, influencia o margen de autonomía, sentido del trabajo, inseguridad sobre el empleo, inseguridad sobre las condiciones de trabajo, apoyo social de los compañeros, claridad de rol, apoyo social de los superiores, previsibilidad, calidad de liderazgo y justicia - estima.

Se observó deficiencias en el liderazgo, siendo uno de los principales factores de riesgo psicosocial y si bien en los niveles de respuesta obtenidos de la unidad hospitalaria no llegan a convertirse totalmente en desfavorables, tampoco existen resultados favorables ni en ésta, ni sobre otras variables de estudio.

Si bien la investigación muestra que los trabajadores del hospital básico Píllaro tienen un desempeño laboral aceptable o es el esperado, muy pocos alcanzan niveles excelentes o mejor al esperado.

Los resultados muestran que el caso de estudio no dispone de guías y/o herramientas técnicas, así como tampoco personal capacitado en el estudio de riesgos psicosociales que contribuya a disminuir los niveles de exposición psicosocial encontrados en los colaboradores.

Se requiere la elaboración de un manual o guía de apoyo que permita a los responsables evaluar, medir e interpretar el desempeño laboral con una adecuada toma de decisiones. 


\section{Referencias}

Acevedo, G. (2013). Riesgos Psicosociales en el Equipo de Salud de Hospitales Públicos de la Provincia de Córdoba, Argentina. Obtenido de https://scielo.conicyt.cl/pdf/cyt/v15n48/art07.pdf

Dirección Distrital 18D05-Santiago de Pillaro - Salud. (2021). Dirección Distrital 18D05-Santiago de Pillaro Salud. Obtenido de https://ecuadornegocios.com/info/direccion-distrital-18d05-santiago-de-pillaro-salud-4410333

Dirección Provincial de Salud de Tungurahua. (2021). Dirección Provincial de Salud de Tungurahua. Obtenido de http://instituciones.msp.gob.ec/dps/tungurahua/index.php/component/content/frontpage

Gil, P. (2012). Riesgos psicosociales en el trabajo y salud ocupacional. Obtenido de http://www.scielo.org.pe/ scielo.php?pid=s1726-46342012000200012\&script=sci_arttext

Hospital Básico Píllaro. (2021). Hospital Básico Píllaro . Obtenido de https://www.dateas.com/en-us/explore/ establecimientos-salud-ecuador/hospital-basico-de-pillaro-1939

Instituto Sindical de Trabajo, Ambiente y Salud de España. (2020). Instituto Sindical de Trabajo, Ambiente y Salud de España. Obtenido de https://istas.net/

Ministerio de Salud Pública. (2021). Ministerio de Salud Pública. Obtenido de https://www.salud.gob.ec/

Organización Mundial de la Salud . (2017). Organización Mundial de la Salud . Obtenido de https://www.who. int/es

Red Regional de Bibliotecas OIT para América Latina y el Caribe. (2017). Red Regional de Bibliotecas OIT para América Latina y el Caribe. Obtenido de https://www.ilo.org/americas/lang--es/index.htm

Ruiz, L. (2021). Alfa de Cronbach (a): qué es y cómo se usa en estadística. Obtenido de https://psicologiaymente. com/miscelanea/alfa-de-cronbach

Salgado, A. C. (2007). Investigación cualitativa: diseños, evaluación del rigor metodológico y retos. Obtenido de http://www.scielo.org.pe/scielo.php?script=sci_arttext\&pid=S1729-48272007000100009

Secretaría Nacional Técnica de Desarrollo de Recursos Humanos y Remuneración del Sector Público. (2007). Secretaría Nacional Técnica de Desarrollo de Recursos Humanos y Remuneración del Sector Público. Obtenido de http://www.trabajo.gob.ec/wp-content/uploads/downloads/2012/11/2007-155-POLITICASESTRATEGIAS-QUE-PERMITEN-A-UARHS-GESTIONAR-CON-MEF.pdf 